\title{
Medical Research During the COVID-19 Pandemic - Two Faces of the Same Coin
}

\author{
Diana Opincariu \\ "George Emil Palade" University of Medicine, Pharmacy, Science and Technology, Târgu Mureș, Romania
}

\section{CORRESPONDENCE}

Diana Opincariu

Str. Gheorghe Marinescu nr. 50

540136 Târgu Mureș, Romania

Tel: +40 372653100

E-mail: diana.opincariu@yahoo.ro

\section{THE RISE OF A GLOBAL HEALTHCARE PROBLEM}

The continuous growth of infections related to the novel coronavirus has affected the healthcare system and public health across the world. The World Health Organization (WHO) has declared COVID-19 outbreak a pandemic on March 11, 2020, when there were more than 20,000 patients with confirmed infections across Europe and the death toll has reached an estimated number of 1,000 people. $^{1}$ This is the first pandemic caused by a coronavirus, and the number of infected subjects, associated mortality rates, and the number of affected countries are expected to rise rapidly. ${ }^{2}$ Therefore, several countries have started to implement national emergency measures to limit and mitigate the spread of infection. At this time, it is of utmost importance to provide sufficient resources to maintain the proper functioning of healthcare systems across the world.

The associated restrictions and measures are to be established at national levels, according to the resources and capabilities of each country. However, WHO strongly recommends the implementation of self-isolation measures, quarantines, and social distancing as soon as possible in order to flatten the curve of infection. The preventive and restrictive actions taken by governments may delay the spread of the virus, thus offering healthcare systems time to allocate the needed logistics for a smooth assimilation of the COVID-19 impact. ${ }^{3}$

As this unparalleled public health crisis is on the rise, it will distress not only the infected subjects and healthcare systems, but it will also take its toll on all societal sectors and individuals, including the economy, research, and industry. Therefore, there is an acute need for urgent research on all aspects of COVID-19 contagion, from genome sequencing and molecular studies, to observational ones that can better characterize the clinical characteristics of this novel disease, with its related complications, morbidity, and mortality. The relatively scarce information available on this pandemic includes the therapeutic measures as well. Currently, there are no proven effective and safe antiviral treatments, and hospitals across the globe are practicing emergency care and empirical treatments for the critically ill, as well as off-label medications that have been used for other 
infectious diseases such as hydroxychloroquine or antivirals established for treating the influenza virus and HIV. ${ }^{4}$

\section{CLINICAL RESEARCH DURING THE COVID-19 PUBLIC HEALTH EMERGENCY}

In medical research during the COVID-19 era, there are two sides of the same coin, which should be preferably conducted in tandem. One side consists in continuing the pre-pandemic clinical trials using the main principles of a qualitative research, with a minimum delay in releasing safe and effective medications on the market. The other side of the coin consists in developing new research that addresses the crisis. Much of the resources allocated for medical research has been shifted towards COVID-19, with a focus on trials that have a large impact over a short amount of time. There is an immense pressure on the scientific community to conduct rapid, effective studies on COVID-19, and especially on treatment methods. ${ }^{5}$ Such conduct may lead to decreased rigors in regards to research methodology, data generation, and interpretation, which may have deleterious effects on a global scale. The rapid conduction of clinical trials for effective treatments and vaccine against COVID-19 may lead to over- or misinterpretation of results. These may disseminate fast via lay media channels, and ultimately become endorsed by governments. ${ }^{6}$ This will not only lead to a false sense of efficacy, but also to a redistribution of resources, away from other, promising studies. Despite the urgent need for new research and evidence on treatments and vaccines for COVID-19, the quality of studies should not be overlooked, as there is a risk for disseminating inaccurate data that can give false leads and ineffective and unsafe practices.

\section{CLINICAL STUDIES - WORKING AROUND THE PROBLEM}

The novel coronavirus pandemic has triggered important disruptions on the development and implementation of new clinical trials. It seems that this interruption will be prolonged as long as there is community contagion risk and circulation restrictions. This is of particular importance in medical areas that depend on experimental trials and testing of new drugs, especially in cancer research, but also in cardiovascular medicine. The short-term modifications triggered by the pandemic are caused by the reallocation of human and financial resources to COVID-19-supporting hospitals, and also cessation of research activities in academic institutions and university hospitals. This will ultimately lead to long-term effects including delayed drug development and testing, thus negatively affecting patient outcomes. ${ }^{7}$ Protocol deviations and impossibility to initiate new medication trials will not only affect future therapeutic managements, but will also come with detrimental financial implications. In addition, there may be a delay or lack in reporting adverse events in clinical trials for medical and pharmacological products, which could affect patient safety on the long term. There should also be a very robust and accurate report in case of infection or COVID19-related deaths of study participants, as it can affect survival end-points in certain studies. ${ }^{8}$

\section{GUIDANCE RECOMMENDATIONS FOR ONGOING CLINICAL TRIALS DURING THE COVID-19 PANDEMIC}

The COVID-19 outbreak at a worldwide level comes with subsequent alterations of ongoing clinical trials. Several measures taken by the authorities for decreasing contagion and increasing healthcare efficacy will lead to study protocol deviations. Additional challenges may result from closure of sites, travel limitations, and interruptions in the supply chain of the tested medical product, but also the infection of study subjects and site investigators. Such protocol deviations should be documented and preferably not impact the future results of current trials. In order to ensure a minimum disruption in the integrity of clinical trials during the SARS-CoV2 pandemic, the Food and Drug Administration (FDA) and the European Medicines Agency (EMA) have both issued guidance documents for the management of ongoing studies of medical products. Both guideline documents have a common clear goal to assure patient safety with a minimum to null risk for infection, while complying with Good Clinical Practices. ${ }^{9,10}$ If the safety of study participants and investigators is put at risk, the proposed measures can go to such lengths as to temporarily cease recruitment and prioritize critical activities for the already enrolled subjects. For experimental drug trials, due to the required social distancing and limitation of outdoor activities, one option could include shipments of study medications and products to patients' homes. However, this approach is feasible in case of study interventions that can be self-administered and do not require in-hospital settings and monitoring. Also, study products should be transported in accordance with the labelled storage conditions, with thorough records of storage, transportation, and destructions of the drug. Another proposed safety measure refers to patient monitoring and study visits, which should be reorganized by using remote electronic and telephone visit protocols. Some presched- 
uled visits are crucial in assessing patient safety, or require imaging or laboratory analysis. If the patient is exposed to unacceptable risk, the recommended option is to either discontinue study participation, or to refer the patient to an alternative lower risk study site. Anyhow, the recommended strategy is to implement alternative processes for study procedures, visits, and supplying the study products in a manner as consistent as possible to the original protocol, in order to ensure a minimum disruption in finalizing the research, while eliminating hazards and COVID-19 exposure. ${ }^{8-10}$

\section{MAJOR TRIALS FOR REPURPOSING ESTABLISHED THERAPEUTIC AGENTS TOWARDS CORONAVIRUS}

There are a multitude of interventional clinical studies that have been initiated in an attempt to provide safe and effective therapeutic options for COVID-19 infection. Two large multicenter studies have been approved for conducting a comparative analysis between several treatments used for other conditions, which have also been used offlabel in COVID-19 patients. Both trials aim to generate robust information regarding the most effective therapeutic strategies for COVID-19. The Solidarity international trial provides a flexible, simplified research platform for testing 4 existing anti-inflammatory and antiviral agents (remdesivir vs. lopinavir-ritonavir, lopinavir-ritonavir combined with interferon-beta vs. hydroxychloroquine) in comparison to local standards of care. The simplified study protocol and flexibility will enable study sites to provide the complete required study data, even in overloaded hospitals that are designated to treat coronavirus patients. ${ }^{11}$ The RECOVERY trial (Randomised Evaluation of COVID-19 Therapy) will comparatively study the effect of 4 potential treatments for COVID-19 infection (lopinavir-ritonavir vs. low-dose dexamethasone vs. hydroxychloroquine vs. tocilizumab). As data from the trial will emerge, the intermediate results will be made public, and any effective treatment will be made available for all patients. Study end-points for RECOVERY include in-hospital death, discharge, and the need for ventilation. The innovative characteristic of this study will be that, if there is a new promising therapeutic agent, it will be included in the study, in addition to the already included medications. ${ }^{12}$

\section{CONCLUSIONS}

The rapid spread of COVID-19 across the world has impacted all aspects of life. The need for effective and safe therapies is acute and can only be established by conducting flexible, simple randomized clinical trials. However, the quality and methodology of medical research is not to be dismissed. Trial misconducts can have a huge impact on the evolution of this pandemic, on a global scale, and they may also lead to improper allocation of financial and human resources. In addition to shifting the research interest and funds towards COVID-19, the ongoing clinical trials should not be neglected. Study procedures and visits should limit the risk of infection for the study participants. If the safety of the subject is put at risk, temporary or permanent cessation of the study should be taken into account. The COVID-19 pandemic is currently dominating all aspects of medical research and healthcare resources. The longer its spread across time, the more obstacles will appear during the aftermath. This will completely change the world of clinical research and will impact future results of studies and trials, in all medical areas.

\section{CONFLICT OF INTEREST}

Nothing to declare.

\section{REFERENCES}

1. WHO announces COVID-19 outbreak a pandemic. Available at: http://www. euro.who.int/en/health-topics/health-emergencies/coronavirus-covid-19/ news/news/2020/3/who-announces-covid-19-outbreak-a-pandemic

2. Li H, Liu SM, Yu XH, Tang SL, Tang CK. Coronavirus disease 2019 (COVID-19): current status and future perspectives. Int J Antimicrob Agents. 2020;105951.

3. Coronavirus disease (COVID-19) Pandemic. Available at: https://www.who. int/emergencies/diseases/novel-coronavirus-2019

4. Guo YR, Cao QD, Hong ZS, et al. The origin, transmission and clinica therapies on coronavirus disease 2019 (COVID-19) outbreak - an update on the status. Mil Med Res. 2020;7:11.

5. lacobucci G. Covid-19 makes the future of UK clinical research uncertain BMJ. 2020;369:m1619.

6. Kim AHJ, Sparks JA, Liew JW, et al. A Rush to Judgment? Rapid Reporting and Dissemination of Results and Its Consequences Regarding the Use of Hydroxychloroquine for COVID-19. Ann Intern Med. 2020;M20-1223.

7. European Centre for Disease Prevention and Control. Outbreak of novel coronavirus disease 2019 (COVID-19): increased transmission globally - fifth update, ECDC: Stockholm; 2020. Available at: https://www.ecdc. europa.eu/sites/default/files/documents/RRA-seventh-update-Outbreakof-coronavirus-disease-COVID-19.pdf (27 March 2020)

8. Medicines and Healthcare products Regulatory Agency. Managing clinical trials during Coronavirus (COVID-19). Available at: https://www.gov.uk/ guidance/managing-clinical-trials-during-coronavirus-covid-19

9. European Medicines Agency. Guidance to sponsors on how to manage clinical trials during the COVID-19 pandemic. Available at: https://www. ema.europa.eu/en/documents/press-release/guidancesponsors-howmanage-clinical-trials-during-covid-19-pandemic_en.pdf (29 March, 2020).

10. US FDA. FDA guidance on conduct of clinical trials of medical products during COVID-19 pandemic. Available at: https://www.fda.gov/ media/136238/download

11. Solidarity trial. Available at: https://www.who.int/emergencies/diseases/ novel-coronavirus-2019/global-research-on-novel-coronavirus-2019ncov/solidarity-clinical-trial-for-covid-19-treatments

12. RECOVERY trial. Available at: https://www.recoverytrial.net 